\title{
Correction to: The Italian validation of the communicative effectiveness index questionnaire: a multicentric study
}

\author{
Pasquale Moretta ${ }^{1}$ Anna Lanzillo ${ }^{1} \cdot$ Maria Daniela Lo Sapio ${ }^{1} \cdot$ Simona Spaccavento $^{2}$ - Fara Cellamare ${ }^{2}$. \\ Francesca Nisoli ${ }^{3}$ - Alessandra lanni ${ }^{3} \cdot$ Debora Pain $^{4} \cdot$ Sarah Feroldi ${ }^{4} \cdot$ Chiara Angela Forlani $^{5} \cdot$ Sara Boccola $^{5}$. \\ Francesca Madonia ${ }^{6} \cdot$ Paolo Volanti $^{6}$. Antonia Pierobon ${ }^{7}$ - Ilaria Scola ${ }^{7}$ - Marina Manera ${ }^{8} \cdot$ Giorgio Bertolotti $^{3}$. \\ Luigi Trojano ${ }^{9}$
}

Published online: 15 April 2021

(C) Fondazione Società Italiana di Neurologia 2021

\section{Correction to: Neurological Sciences (2021) https://doi.org/10.1007/s10072-020-04781-z}

The original article contains an error in author name. The author "Antonia Pierobon" was incorrectly spelled as "Antonia Pierbon. The correct author name is presented above.
The original article has been corrected.

Publisher's note Springer Nature remains neutral with regard to jurisdictional claims in published maps and institutional affiliations.
The online version of the original article can be found at https://doi.org/ 10.1007/s10072-020-04781-Z

Pasquale Moretta

pasquale.moretta@icsmaugeri.it

Istituti Clinici Scientifici Maugeri IRCCS, Department of Neurorehabilitation, Institute of Telese Terme, Benevento, Italy

2 Istituti Clinici Scientifici Maugeri IRCCS, Department of Neurorehabilitation, Institute of Bari, Bari, Italy

3 Istituti Clinici Scientifici Maugeri IRCCS, Department of Neurorehabilitation, Institute of Tradate, Milan, Italy

4 Istituti Clinici Scientifici Maugeri IRCCS, Department of Neurorehabilitation, Institute of Milano, Milan, Italy
Istituti Clinici Scientifici Maugeri IRCCS, Department of Neurorehabilitation, Institute of Castel Goffredo, Mantova, Italy

6 Istituti Clinici Scientifici Maugeri IRCCS, Department of Neurorehabilitation, Institute of Mistretta, Messina, Italy

7 Istituti Clinici Scientifici Maugeri IRCCS, Department of Neurorehabilitation, Institute of Montescano, Pavia, Italy

8 Istituti Clinici Scientifici Maugeri IRCCS, Department of Neurorehabilitation, Institute of Pavia, Pavia, Italy

9 Department of Psychology, University of Campania Luigi Vanvitelli, Caserta, Italy 